\title{
Switchable coupling for superconducting qubits using double resonance in the presence of crosstalk
}

\author{
S. Ashhab ${ }^{1}$ and Franco Nori ${ }^{1,2}$ \\ ${ }^{1}$ Frontier Research System, The Institute of Physical and Chemical Research (RIKEN), Wako-shi, Saitama 351-0198, Japan \\ ${ }^{2}$ Physics Department and Michigan Center for Theoretical Physics, \\ The University of Michigan, Ann Arbor, Michigan 48109-1040, USA
}

(Dated: October 31, 2018)

\begin{abstract}
Several methods have been proposed recently to achieve switchable coupling between superconducting qubits. We discuss some of the main considerations regarding the feasibility of implementing one of those proposals: the double-resonance method. We analyze mainly issues related to the achievable effective coupling strength and the effects of crosstalk on this coupling mechanism. We also find a new, crosstalk-assisted coupling channel that can be an attractive alternative when implementing the double-resonance coupling proposal.
\end{abstract}

Superconducting systems are among the leading candidates for the implementation of quantum information processing applications [1]. In order to perform multiqubit operations, one needs a reliable method for switchable coupling between the qubits, i.e. a coupling mechanism that can be easily turned on and off. Over the past few years, there has been considerable interest in this question, both theoretically [2, 3, 4, 5, 6, 7] and experimentally [8, 9].

The most obvious approach for switchable coupling is probably the one based on the application of dc pulses [2]. However, given the difficulty of achieving accurate dc pulses and complications associated with taking the qubits away from their low-decoherence degeneracy points, recent efforts have focused on proposals using fixed dc bias conditions, with the coupling being turned on and off using ac signals [3, 4, 5, 5, 6, 7]. The first such proposal [3] uses resonant oscillating fields applied to the two qubits and employs ideas from the double-resonance concept in NMR [10]. The method was generalized in Ref. 7] to relax the condition of resonant driving on the two qubits. Relaxing the resonant-driving requirement implies relaxing the requirement of strong driving fields, a requirement that is undesirable in superconducting qubit systems.

There is currently intense experimental effort to demonstrate switchable coupling between superconducting qubits. Although the apparently simple implementation of the double-resonance method makes it an attractive option to use, a number of relevant questions remain unanswered. In this paper we analyze some considerations that would be useful to an experimentalist attempting to implement it. We discuss mainly (1) the relation between the strength of the applied fields and the resulting effective coupling strength and (2) the possibility of reducing or eliminating crosstalk effects using slow turn-on and turn-off of the applied fields. The first question is important for deciding the optimal driving parameters to use while taking into consideration the limited decoherence times and limitations on the strong driving of the qubits. In connection with the second question, we find an alternative coupling channel that is easier to drive and results in smoother oscillations.

Switchable coupling using double resonance - We first review, with a minor generalization, the derivation of the double-resonance coupling mechanism of Ref. [7]. We consider a system composed of two qubits with fixed bias and interaction parameters. Oscillating external fields can then be applied to the system in order to perform the different gate operations.

The effective Hamiltonian of the system is given by:

$$
\hat{H}=-\sum_{j=1}^{2}\left(\frac{\omega_{j}}{2} \hat{\sigma}_{z}^{(j)}+\Omega_{j} \cos \left(\omega_{j}^{\mathrm{rf}} t+\varphi_{j}\right) \hat{\sigma}_{x}^{(j)}\right)+\frac{\lambda}{2} \hat{\sigma}_{x}^{(1)} \hat{\sigma}_{x}^{(2)}
$$

where $\omega_{j}$ is the Larmor frequency of the qubit labelled with the index $j ; \Omega_{j}, \omega_{j}^{\mathrm{rf}}$ and $\varphi_{j}$ are, respectively, the amplitude, frequency and phase of the applied oscillating fields; $\lambda$ is the inter-qubit coupling strength; and $\hat{\sigma}_{\alpha}^{(j)}$ are the Pauli matrices with $\alpha=x, y, z$ and $j=1,2$ (note that we have set $\hbar=1)$. The eigenstates of $\hat{\sigma}_{z}$ are denoted by $|g\rangle$ and $|e\rangle$, with $\hat{\sigma}_{z}|g\rangle=|g\rangle$.

As discussed in Ref. [7], we also take $\lambda \ll \Delta \ll \omega$, where $\Delta=\omega_{1}-\omega_{2}$ (we take $\omega_{1}>\omega_{2}$ and $\lambda>0$ with no loss of generality), and $\omega$ represents the typical size of the parameters $\omega_{j}$.

We now show how one can drive oscillations between the states $|g g\rangle$ and $|e e\rangle$ using the double-resonance method. We take the Hamiltonian in Eq. (11) and transform it using the unitary operation $\hat{S}_{1}(t)=$ $\exp \left\{i \sum_{j=1}^{2}\left(\omega_{1}^{\mathrm{rf}} \hat{\sigma}_{z}^{(1)}+\omega_{2}^{\mathrm{rf}} \hat{\sigma}_{z}^{(2)}\right) t / 2\right\}$, such that we obtain the transformed Hamiltonian $\hat{H}^{\prime}=\hat{S}_{1}^{\dagger}(t) \hat{H} \hat{S}_{1}(t)-$ $i \hat{S}_{1}^{\dagger}\left(d \hat{S}_{1} / d t\right)$. Performing a rotating-wave approximation (RWA), we obtain:

$$
\hat{H}^{\prime}=-\sum_{j=1}^{2}\left(\frac{\delta \omega_{j}}{2} \hat{\sigma}_{z}^{(j)}+\frac{\Omega_{j}}{2}\left[\cos \varphi_{j} \hat{\sigma}_{x}^{(j)}-\sin \varphi_{j} \hat{\sigma}_{y}^{(j)}\right]\right)
$$




$$
\begin{aligned}
& +\frac{\lambda}{4}\left(\hat{\sigma}_{x}^{(1)} \hat{\sigma}_{x}^{(2)} \cos \delta \omega_{\mathrm{rf}} t+\hat{\sigma}_{y}^{(1)} \hat{\sigma}_{y}^{(2)} \cos \delta \omega_{\mathrm{rf}} t\right. \\
& \left.+\hat{\sigma}_{y}^{(1)} \hat{\sigma}_{x}^{(2)} \sin \delta \omega_{\mathrm{rf}} t-\hat{\sigma}_{x}^{(1)} \hat{\sigma}_{y}^{(2)} \sin \delta \omega_{\mathrm{rf}} t\right)
\end{aligned}
$$

where $\delta \omega_{j}=\omega_{j}-\omega_{j}^{\mathrm{rf}}$, and $\delta \omega_{\mathrm{rf}}=\omega_{1}^{\mathrm{rf}}-\omega_{2}^{\mathrm{rf}}$. We now perform a basis transformation in spin space from the operators $\hat{\sigma}$ to the operators $\hat{\tau}$ such that the time-independent terms in Eq. (2) are parallel to the new $z$ axes and the new $y$ axes lie in the old $x-y$ planes (note here that we are performing two different transformations for the two qubits). Following the same steps as in Ref. [7] while choosing driving parameters that satisfy the resonance condition

$$
\sqrt{\delta \omega_{1}^{2}+\Omega_{1}^{2}}+\sqrt{\delta \omega_{2}^{2}+\Omega_{2}^{2}}=\omega_{1}^{\mathrm{rf}}-\omega_{2}^{\mathrm{rf}}
$$

we reach the effective Hamiltonian

$$
\hat{H}^{\prime \prime}=-\frac{\lambda_{\text {eff }}}{2}\left\{e^{i \delta \varphi} \hat{\tau}_{+}^{(1)} \hat{\tau}_{+}^{(2)}+e^{-i \delta \varphi} \hat{\tau}_{-}^{(1)} \hat{\tau}_{-}^{(2)}\right\}
$$

where we have defined

$$
\lambda_{\text {eff }}=\frac{\lambda}{4}\left(1-\cos \theta_{1}\right)\left(1+\cos \theta_{2}\right),
$$

$\delta \varphi=\varphi_{1}-\varphi_{2}, \tau_{ \pm}^{(j)}=\left(\tau_{x}^{(j)} \pm i \tau_{y}^{(j)}\right) / 2$, and $\tan \theta_{j}=$ $\Omega_{j} / \delta \omega_{j}$. Alternative resonance conditions are given by $\pm \sqrt{\delta \omega_{1}^{2}+\Omega_{1}^{2}} \mp \sqrt{\delta \omega_{2}^{2}+\Omega_{2}^{2}}=\left|\omega_{1}^{\mathrm{rf}}-\omega_{2}^{\mathrm{rf}}\right|$, and they result in simple variations of the effective Hamiltonian given in Eq. (41) [11].

Equations (3) and (4) form the basis for the doubleresonance coupling mechanism. The Hamiltonian $\hat{H}^{\prime \prime}$ drives the transition $|g g\rangle \leftrightarrow|e e\rangle$ but does not affect the states $|g e\rangle$ and $|e g\rangle$ in the basis of the operators $\hat{\tau}$. Therefore, a single two-qubit gate that can be performed using the Hamiltonian $\hat{H}^{\prime \prime}$ and the set of all single-qubit transformations form a universal set of gates for quantum computing.

Effective coupling strength - The first question we consider here is the relation between the strength of the driving fields and the effective coupling strength, i.e. the two-qubit gate speed. Equation (3) can be satisfied with values of $\Omega_{j}$ ranging from 0 to much higher than $\Delta$, although our second RWA breaks down for those large values (the deviations from the above derivation should be fixable by including higher-order effects, as long as the driving amplitudes remain much smaller than the qubit Larmor frequencies).

First let us look at the meaning of the coupling strength $\lambda$. If the two qubits were exactly resonant with each other $\left(\omega_{1}=\omega_{2}\right)$, two-qubit oscillations occur with (angular) frequency $\lambda$. Although we are unaware of any mathematical proof in the literature, it seems to us intuitively obvious that this value should be the upper bound of how fast one can perform two-qubit operations utilizing this coupling term. The ratio $\lambda_{\text {eff }} / \lambda$ therefore tells us

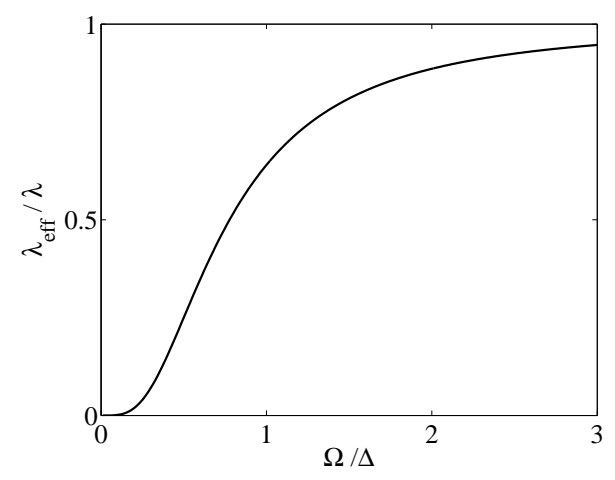

FIG. 1: The effective coupling strength $\lambda_{\text {eff }}$ divided by the original coupling strength $\lambda$ from Eq. (1) as a function of the driving amplitude $\Omega$ divided by the inter-qubit detuning $\Delta$. Here we assume equal driving amplitudes for the two qubits.

how much of the maximum speed we are achieving with a given set of driving parameters. This ratio is given by:

$$
\frac{\lambda_{\mathrm{eff}}}{\lambda}=\frac{1}{4}\left[1+\frac{x-\frac{1}{4 x}}{\sqrt{1+\left(x-\frac{1}{4 x}\right)^{2}}}\right]^{2},
$$

where $x=\Omega / \Delta$, and we have assumed equal driving amplitudes for both qubits. The ratio $\lambda_{\text {eff }} / \lambda$ is plotted in Fig. 1. For weak driving fields, $\lambda_{\text {eff }} / \lambda$ is proportional to $\Omega^{4}$, making it highly undesirable to go too far into that limit.

It might seem strange that it is possible to achieve the full speed allowed by the coupling term in Eq. (1) [see Fig. 1 with $\Omega / \Delta \rightarrow \infty$ ], a situation intuitively reserved for the case of resonant qubits. Inspection of the resonance condition (Eq. 3) in this strong-driving limit shows that it corresponds to the driving fields being far off resonance with the qubits and

$$
\omega_{1}+\frac{\Omega_{1}^{2}}{2\left(\omega_{1}-\omega_{1}^{\mathrm{rf}}\right)}=\omega_{2}+\frac{\Omega_{2}^{2}}{2\left(\omega_{2}-\omega_{2}^{\mathrm{rf}}\right)} .
$$

The effect of the driving fields can therefore be understood in terms of the ac-Stark shifts added to the qubit Larmor frequencies. The resonance condition is satisfied when the renormalized Larmor frequencies of the two qubits are equal. From this point of view, it is not surprising that one can achieve the full speed allowed by the coupling term [12]. The experiment of Ref. [9] was close to this limit.

For any realistic situation, our approximations will break down before achieving the infinitely strong driving limit. The relationship between the different parameters determines how close one can get to that limit. With the typical parameters $\omega_{1} / 2 \pi=5 \mathrm{GHz}, \Delta / 2 \pi=1 \mathrm{GHz}$ and $\lambda / 2 \pi=0.1 \mathrm{GHz}$, we numerically obtain $70-80 \%$ of the maximum gate speed before the dynamics deviates from that of Eq. (41) [in all our numerical calculations, we solve the Schrödinger equation using the Hamiltonian 
in Eq. (1). Here we also use the resonance condition in Eq. (3)].

Crosstalk - We now address the question of crosstalk, i.e. when each qubit feels the driving signal intended for the other qubit. In order to describe the effects of crosstalk, we now add to the Hamiltonian in Eq. (11) terms of the form $\beta \Omega_{j} \cos \left(\omega_{j}^{\mathrm{rf}} t+\varphi_{j}\right) \sigma_{x}^{\left(j^{\prime}\right)}$, where $j \neq j^{\prime}$, and the coefficient $\beta$ quantifies the amount of crosstalk. As the presently relevant case for flux qubits, we take $\beta=1$ (i.e., $100 \%$ crosstalk).

In order to understand the harmful effects of crosstalk [13], one should note that the Hamiltonian in Eq. (44) does not operate in the computational basis, but rather in a rotated basis. Reference [3] proposed using a precise timing procedure where meaningful results are obtained only at times when the two bases coincide, whereas Ref. [7] proposed applying the appropriate single-qubit rotations before and after the two-qubit gate operation. Both proposals add a step to the coupling procedure, and precise calibration is required for this extra step. Corrections from crosstalk must therefore be taken into account in implementing the extra step.

Here we propose to turn the driving signal on and off slowly, such that the adiabatic ramp of the driving signal's amplitude transforms the states of the qubits between the computational basis and the rotated basis of Eq. (44). With this approach the extra steps mentioned above are no longer needed. Clearly, and especially from an experimental point of view, eliminating a step from the required procedure is always a welcome simplification, regardless of crosstalk. With our new proposal, one would need to adjust only one parameter in order to obtain good oscillation dynamics.

Since the idea of slowly turning on and off nearresonant driving fields has not been analyzed in the literature for this system, we discuss it a little bit further here. We consider a single qubit. We would like to take a qubit in an arbitrary quantum state and adiabatically transform the quantum state from the computational basis into the dressed-state basis, leaving the form of the quantum state unaffected (note that the discussion of this system is similar to that of a spin- $1 / 2$ particle in a changing magnetic field). For a moment, we take the frequency of the driving field to be fixed and the amplitude increasing from zero to its final value. In this case we are effectively dealing with a rotating-frame field that starts along the $z$ axis with initial value $|\delta \omega|=\left|\omega-\omega^{\mathrm{rf}}\right|$ and changes (with the additional component in the $x-y$ plane) to the final value $\sqrt{\delta \omega^{2}+\Omega^{2}}$. In order to maintain adiabaticity, the timescale over which we have to turn on the driving fields must be larger than $1 /|\delta \omega|$, since the initial point is the most susceptible to non-adiabatic transitions. In particular, note that in the case of resonant driving (i.e., $\delta \omega=0$ ), it is impossible to turn on the field adiabatically in the above sense. The main limitation imposed by this adiabatic-turn-on approach is therefore that it cannot be used with parameters that are too close to the resonant-driving case $(\delta \omega=0)$.
In the previous paragraph we have established the upper bound for the allowed speed of turning on the driving fields, dictated by single-qubit adiabaticity requirements. We now note that, in order to maintain the switchable nature of the coupling mechanism, the process of turning on the driving field must be fast compared to the timescale of two-qubit oscillations. Using the same parameters as above $\left(\omega_{1} / 2 \pi=5 \mathrm{GHz}, \Delta / 2 \pi=1 \mathrm{GHz}\right.$ and $\lambda / 2 \pi=0.1 \mathrm{GHz}$ ), we find that $|\delta \omega|$ must be substantially larger than $25 \mathrm{MHz}$ in order to be able to satisfy both requirements. If we take $|\delta \omega|$ to be a few hundred $\mathrm{MHz}$, we find that we have a large enough window of values for the turn-on time such that this process is slow with respect to the single-qubit dynamics, but fast with respect to the two-qubit dynamics.

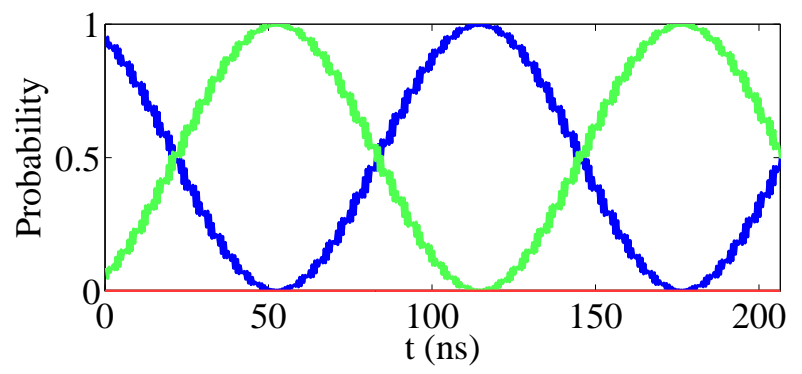

FIG. 2: (color online) The occupation probabilities of the four computational states as functions of time. The black, blue (gray), green (light-gray) and red (dark-gray) lines (the black and red lines are indistinguishable from the $x$ axis) correspond, respectively, to the states $|g g\rangle,|g e\rangle,|e g\rangle$ and $|e e\rangle$. The initial state is $|g e\rangle, \omega_{1} / 2 \pi=5 \mathrm{GHz}, \omega_{2} / 2 \pi=4 \mathrm{GHz}, \lambda / 2 \pi=0.1$ $\mathrm{GHz}$, and $\delta \omega_{1}=-\delta \omega_{2}=0.15 \mathrm{GHz}$. The amplitudes of the driving fields were adjusted to reach optimal results, keeping $\Omega_{1}=\Omega_{2}$. The driving fields are turned on and off over durations of $20 \mathrm{~ns}$ (note that the time of turning on and off the driving fields is not included in the time axis). In order to see the reduction in crosstalk effects, this figure can be compared with Fig. 4(b) of Ref. [7].

Figure 2 shows oscillations that characterize a twoqubit gate using driving fields that are turned on and off slowly as explained above. Comparison with Fig. 4(b) of Ref. 7] shows that the crosstalk-induced, noise-like fluctuations are greatly reduced. Note that the oscillations are between the states $|g e\rangle$ and $|e g\rangle$, in contrast to the appearance of Eq. (4). The difference is due to the different single-qubit rotations performed in the pulsed and adiabatic approaches.

Interestingly, crosstalk opens a new possibility for coupling between the qubits. An alternative resonance condition is (approximately) given by

$$
\sqrt{\delta \omega_{1}^{2}+\Omega_{1}^{2}}=\sqrt{\delta \omega_{2}^{2}+\Omega_{2}^{2}}
$$

This can be seen by inspecting Fig. 1 of Ref. 7]. This resonance condition corresponds to oscillations between the states $|g g\rangle$ and $|e e\rangle$, using the adiabatic-ramp approach above [14]. In the absence of crosstalk, one can 
say that the effective matrix element for this coupling channel vanishes, and no coupling occurs even if the resonance condition is satisfied. In the presence of crosstalk, two-qubit oscillations do occur.

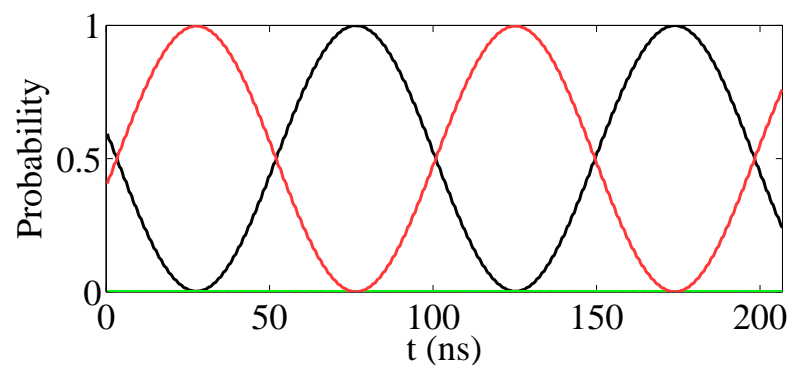

FIG. 3: (color online) Same as in Fig. 2 (the blue and green lines are now indistinguishable from the $x$ axis), but the initial state is $|g g\rangle$ and $\delta \omega_{1}=0.14 \mathrm{GHz}$.

This coupling mechanism can also be treated analytically as above. However, the algebra is now more cumbersome and the results less transparent. The implementation procedure, however, is straightforward. One could for example set the qubit-field detunings $\delta \omega_{1}>0$ and $\delta \omega_{2}<0$, with a small difference between $\delta \omega_{1}$ and $\left|\delta \omega_{2}\right|$. One also sets $\Omega_{1}=\Omega_{2}$ [this choice means that Eq. (8) cannot be satisfied; however, crosstalk shifts modify the resonance condition such that we obtain a resonance peak]. By varying this common amplitude, one can easily locate the resonance. An example of this coupling mechanism is shown in Fig. 3. An important advantage of this approach is that for weak driving the gate speed is higher and the resonance peak is broader in this case than in the traditional case presented above, allowing for more error tolerance using this coupling mechanism (of the order of $5-10 \%$ compared to $0.5 \%$ for typical parameters). The resulting oscillations are also smoother in this case.

Conclusion - We have analyzed some considerations related to the possible experimental implementation of the double-resonance method for achieving switchable coupling between superconducting qubits. We have obtained the dependence of the effective coupling strength on the amplitudes of the applied driving fields. We have also discussed the approach of using slow turn-on and turn-off of the driving fields. This approach reduces, and possibly eliminates, the effects of crosstalk. The two main questions addressed in this paper are important in determining the optimal set of parameters for experimental attempts to implement this coupling mechanism. We have also found that crosstalk opens a new coupling channel that has some advantages over the one used in the original idea of the double-resonance approach.

We would like to thank K. Harrabi, J. R. Johansson, J. Martinis, Y. Nakamura and G. S. Paraoanu for useful discussions. This work was supported in part by the National Security Agency (NSA), the Army Research Office (ARO), the Laboratory for Physical Sciences (LPS), and the National Science Foundation (NSF) grant No. EIA0130383. One of us (S.A.) was supported by the Japan Society for the Promotion of Science (JSPS).
[1] See e.g. J. Q. You and F. Nori, Phys. Today 58 (11), 42 (2005); G. Wendin and V. Shumeiko, in Handbook of Theoretical and Computational Nanotechnology, ed. M. Rieth and W. Schommers (ASP, Los Angeles, 2006).

[2] Y. Makhlin et al., Nature 398, 305 (1999); J. Q. You et al., Phys. Rev. Lett. 89, 197902 (2002); Phys. Rev. B 68, 024510 (2003); D. V. Averin and C. Bruder, Phys. Rev. Lett. 91, 057003 (2003); B. L. T. Plourde et al., Phys. Rev. B 70, 140501(R) (2004).

[3] C. Rigetti et al., Phys. Rev. Lett. 94, 240502 (2005).

[4] Y.-X. Liu et al., Phys. Rev. Lett. 96, 067003 (2006).

[5] P. Bertet et al., Phys. Rev. B. 73, 064512 (2006); A. O. Niskanen et al., Phys. Rev. B 73, 094506 (2006); M. Grajcar et al., Phys. Rev. B 74, 172505 (2006).

[6] G. S. Paraoanu, Phys. Rev. B 74, 140504(R) (2006).

[7] S. Ashhab et al., Phys. Rev. B 74, 184504 (2006).

[8] Yu. A. Pashkin et al., Nature 421, 823 (2003); P. R. Johnson et al., Phys. Rev. B 67, 020509(R) (2003); A. J. Berkley et al., Science 300, 1548 (2003); T. Yamamoto et al., Nature 425, 941 (2003); A. Izmalkov et al., Phys. Rev. Lett. 93, 037003 (2004); H. Xu et al., Phys. Rev. Lett. 94, 027003 (2005); R. McDermott et al., Science 307, 1299 (2005); J. B. Majer et al., Phys. Rev. Lett.
94, 090501 (2005); B. L. T. Plourde et al., Phys. Rev. B 72, 060506(R) (2005); T. Hime et al., Science 314, 1427 (2006); S. H. W. van der Ploeg et al., Phys. Rev. Lett. 98, 057004 (2007); A. O. Niskanen et al., Science 316, 723 (2007); J. Plantenberg et al., Nature 447, 836 (2007); M. A. Sillanpää et al., Nature 449, 438 (2007).

[9] J. Majer et al., Nature 449, 443 (2007).

[10] See, e.g., C. P. Slichter, Principles of Magnetic Resonance (Springer, Berlin, 1996).

[11] We would like to thank M. Devoret, S. Girvin and R. Schoelkopf for pointing out this issue to us.

[12] Note here that in this case $\theta_{2}=\pi$, so that the coupling oscillations occur between the states $|e g\rangle$ and $|g e\rangle$, in spite of the appearance of $\hat{H}_{\text {eff }}$ in Eq. (4).

[13] Some crosstalk effects can, in principle, be eliminated using proper calibration, as was done, e.g., in I. L. Chuang et al., Nature 393, 6681 (1998).

[14] One must also be careful to avoid satisfying this resonance condition if one wishes to use the coupling mechanism mentioned earlier in the manuscript. These crosstalk-assisted oscillations were mentioned, but erroneously dismissed as being harmless, in Ref. [7]. 